\title{
Ultrasound Monitoring of Tissue Ablation Via Deformation Model and Shape Priors
}

\author{
Emad Boctor ${ }^{1}$, Michelle deOliveira ${ }^{2}$, Michael Choti $^{2}$, Roger Ghanem ${ }^{3}$, \\ Russell Taylor ${ }^{1}$, Gregory Hager ${ }^{1}$, and Gabor Fichtinger ${ }^{1}$ \\ ${ }^{1}$ Engineering Research Center, Johns Hopkins University, Baltimore, MD, USA \\ ${ }^{2}$ Department of Surgery, Johns Hopkins Hospital, Baltimore, MD, USA \\ ${ }^{3}$ Department of Aerospace and Mechanical Eng., Univ. of Southern California, CA, USA \\ eboctor@ieee.org
}

\begin{abstract}
A rapid approach to monitor ablative therapy through optimizing shape and elasticity parameters is introduced. Our motivating clinical application is targeting and intraoperative monitoring of hepatic tumor thermal ablation, but the method translates to the generic problem of encapsulated stiff masses (solid organs, tumors, ablated lesions, etc.) in ultrasound imaging. The approach involves the integration of the following components: a biomechanical computational model of the tissue, a correlation approach to estimate/track tissue deformation, and an optimization method to solve the inverse problem and recover the shape parameters in the volume of interest. Successful convergence and reliability studies were conducted on simulated data. Then ex-vivo studies were performed on 18 ex-vivo bovine liver samples previously ablated under ultrasound monitoring in controlled laboratory environment. While Bmode ultrasound does not clearly identify the development of necrotic lesions, the proposed technique can potentially segment the ablation zone. The same framework can also yield both partial and full elasticity reconstruction.
\end{abstract}

\section{Introduction}

Primary and metastatic liver cancer represents a significant source of morbidity and mortality in the United States and worldwide [1]. An increasing interest has been focused on treatment using thermal ablative approaches, particularly radiofrequency ablation (RFA). These approaches utilize image guided placement of a probe within the target area in the liver parenchyma. Heat created around an electrode is conducted into the surrounding tissue, causing coagulative necrosis at a temperature between $50^{\circ} \mathrm{C}$ and $100^{\circ} \mathrm{C}$ [2]. Key problems with this approach include: 1) localization/targeting of the tumor and 2) monitoring of the ablation zone. The first problem has been previously addressed by developing a robotic 3DUS system for guidance of liver ablation. The second problem, the subject of this paper, is monitoring the zone of necrosis during ablative therapy.

Monitoring the ablation process in order to document adequacy of margins during treatment is a significant problem. Current approaches often result in either local failure or excessively large zones of liver ablation. Some ablative devices employ temperature monitoring using thermisters built within the ablation probes. However, these temperatures only provide a crude estimate of the zone of ablation. Magnetic 
resonance imaging (MRI) can monitor temperature changes (MR thermometry), but is expensive, not available in many sites, and difficult to use intraoperatively [3]. Ultrasonography (US) is the most common modality for both target imaging and is also used for ablation monitoring. However, conventional ultrasonographic appearance of ablated tumors only reveal hyperechoic areas due to microbubbles and outgasing, but cannot adequately visualize the margin of tissue coagulation. Currently, ablation adequacy is only estimated at the time of the procedure and primarily based on the probe position and not the true ablation zone.

Accordingly, ultrasound elasticity imaging (USEI), known as elastography, has emerged as a potentially useful augmentation to conventional ultrasound imaging, first introduced by Ophir [11]. USEI in monitoring ablation [4, 13-16] was made possible by the following observations: (1) different tissues may have significant differences in their mechanical properties and (2) the information encoded in the coherent scattering (a.k.a. speckle) may be sufficient to calculate these differences following a mechanical stimulus. However, producing a real-time elasticity map using 3D ultrasound data is an exigent task that requires extensive computation and has its own limitations. Despite the fact that strain images have better signal-to-noise (SNR) and contrast-to-noise (CNR) compared to US images [10, 12] (Fig. 1), these images still suffer from artifacts related to their formation theory or US artifacts. These artifacts are primarily attributable to decorrelation due to out-of-plane motion, large deformation, shadowing effect, or other causes. Moreover, speckle decorrelation occurs due to the shadowing/attenuating effects underneath the hot ablation zone as seen in the Bmode image (Fig. 1, left).

Accurate segmentation of strain images is an essential in planning and monitoring ablations [4, 13-16]. More generally, our method is directly relevant to a large family of interventions that require targeting, tracking, and monitoring some encapsulated stiff mass suspended in a softer background. In this paper, we report a generic and rapid approach to segment stiff lesions based on tissue deformation (i.e. displacements), without needing to estimate strain images.

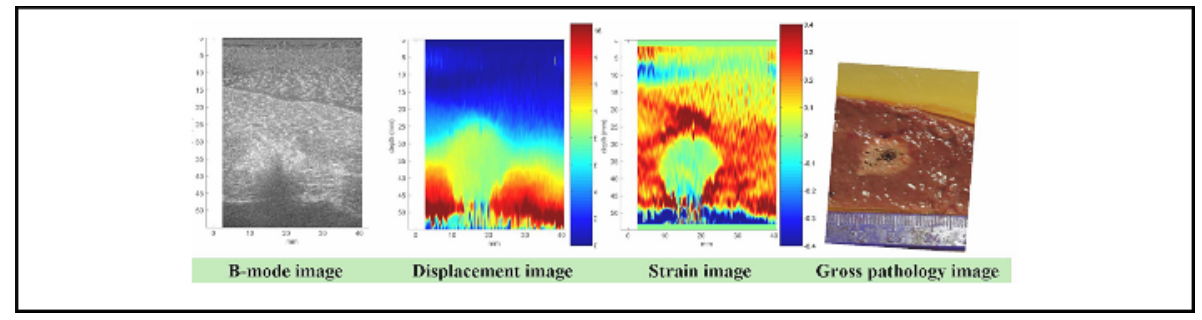

Fig. 1. B-mode image shows ex-vivo liver boundaries embedded in gel based medium. It is not possible to differentiate the ablated area from B-mode. Strain is generated from differentiating a displacement map in the axial direction. Strain provides clear evidence of the presence of hard lesion, which is in agreement with the gross pathology picture.

\section{Strain Imaging Segmentation}

Strain imaging holds the promise of playing a significant role in planning and monitoring ablative therapy [4, 13-16]. However, reliable and rapid semi/automatic 
segmentation of noisy strain images is needed during ablation intervention. By comparing ablated regions in both gross pathology and strain images in the 18 ex-vivo bovine liver samples, strain imaging -immediately after ablation- tends to overestimate ablation coverage in $33.3 \%$ of the time, due to a decorrelation region beneath the ablated lesion $[15,16]$. To appreciate this fact, refer to the strain image in Fig. 1 that has a strong agreement with gross pathology laterally and an obvious overestimation axially. It is only $13.3 \%$ of the time when ablation coverage in strain images matched corresponding gross pathology images. Accurate segmentation should help the surgeon by monitoring the extent of the applied ablative therapy in 3D, planning optimal overlap/multiple ablations to cover the original target tumor, and terminating decision of the procedure.

To extract ablation boundaries, one approach is to apply conventional image segmentation techniques directly to strain images. Initially to get these strain images, we start from RF data as input; create the displacement and correlation images; then differentiate the axial displacement field and estimate the axial strain tensor $(\varepsilon 11)$. Obviously, to have reliable standalone segmentation module, the inputs (strain images) to this module should meet some expected standards, or consistent quality. However, obtaining local strain values with high accuracy depends on precise measurement of local tissue displacement. A significant problem is the loss of similarity (correlation) in the pre- and post-deformed image. During the last few years, several groups have investigated this problem [15] and have came up with various strategies for increasing the reliability of the cross-correlation function including: 1) Choice of the processing parameters, kernel length and amount of kernel overlap [5]; 2) RF-data tracking instead of envelope-detected data when small displacements are involved [6]; 3) Temporal stretching that include adaptive local and global companding [7]; and 4) Axial and lateral RF-data interpolation. In addition to these enhancements for displacement estimation, there has been active research in improving the procedure for deriving strain from displacement images including: 1) A least squares strain estimator (LSQSE) was suggested [8]; 2) Multi-step compression [9] to increase SNR; and 3) Average and median filtering [10].

Besides the perennial problem of decorrelation, the following issues need to be addressed. Displacement estimation: The majority of the rapid techniques assume a constant distribution of scatterers before and after a small ( $<3 \%$ axial strain) introduced axial compression. However, this assumption is not valid during ablative therapy proximal to the ablation zone, where vaporization and bubbles affect speckles appearance and matching. Strain estimation: The procedure for estimating the axial strain tensor is based on differentiating the displacement field. However, differentiation also amplifies errors and noise in the displacement measurements. Unfortunately, all current solutions to suppress noise in strain imaging require least squares estimation or average multiple compression steps. Both add a considerable computation cost and/or time delay. Time performance: The entire chain of displacement estimation, strain image reconstruction, and strain image segmentation takes far too long for 3D datasets. Prior knowledge and redundancy: Clearly, not all displacement/strain measurements are required to locate a lesion. At the same time, in our motivating ablative therapy application, there is well-defined a priori knowledge about the expected shape and size of the ablation. Undoubtedly, utilizing this a priori information effectively should reduce redundant computational cost or time required. Elasticity reconstruction: Segmentation helps in defining the location and boundary of a lesion but 
fails in reconstructing Young's modulus at the region of interest. It will be favorable, if one can augment the segmentation pipeline to solve the inverse problem with minimal effort.

To address all these issues, we propose a novel framework to segment and track ablated lesions based on tissue deformations and shape priors. Furthermore, elasticity reconstruction, i.e. to retrieve the Young's modulus, is possible with minimal alteration of the framework.

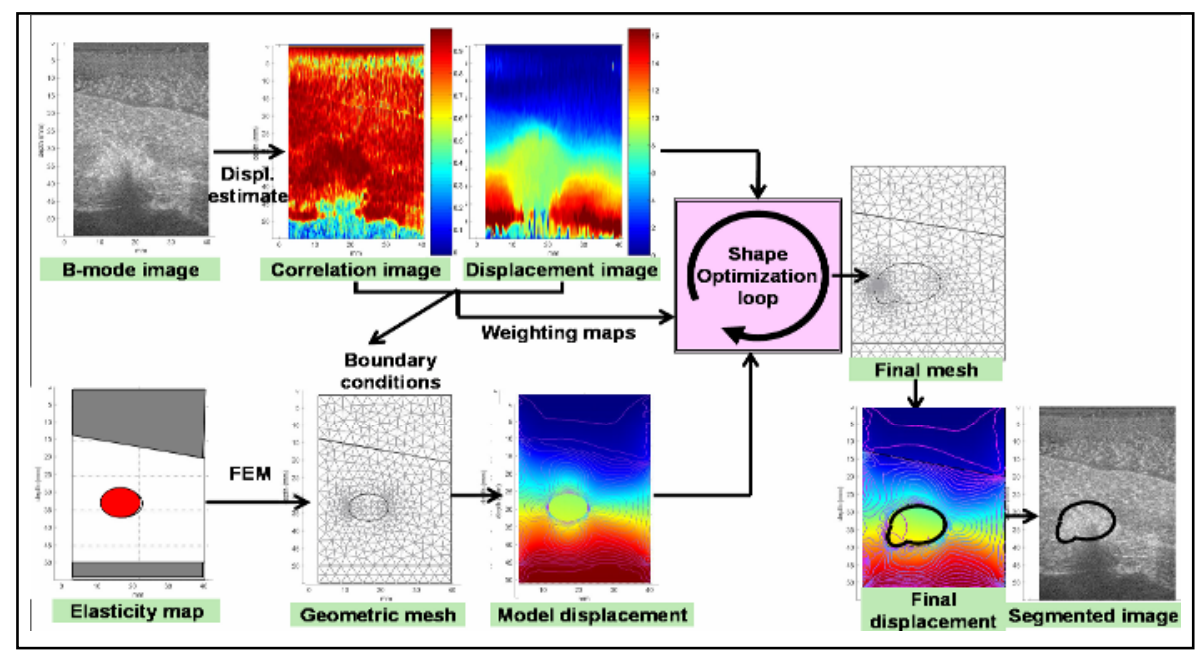

Fig. 2. Iterative tracking and segmentation pipeline

\section{Elasticity Model Based on Tissue Deformations}

The key insight to our approach is the integration of prior geometrical knowledge, a physical elasticity model, and direct estimation of tissue deformation. The proposed approach is explained in Fig. 2. The process starts, in the lower branch of the workflow, from a prior geometrical model. In our example, the ablated lesion is modeled as simple ellipse. In liver ablation, we have a very reliable initial guess of the location of the lesion, because we know the position of the ablator. Next we solve the forward problem of estimating the theoretical displacement from a geometric mesh representation of the initial model, boundary conditions and assumed elasticity model. The computational method of choice to solve this forward problem is Finite Element Method (FEM). In the upper branch, we start with calculating the correlation map and then rapidly estimate the displacement field. The lower and upper branches meet in the shape optimization loop where we iteratively solve the inverse problem to optimize our parametric geometric model and locate the region of interest.

\subsection{Tissue Displacement Estimation}

We acquire RF ultrasound data from a tissue in both rest and stressed states, and then estimate the induced deformation distribution by tracking speckle motion. Our 
implementation is based on maximization of normalized cross-correlation between pre- and post-compressed RF signals. In the example shown in Figure 2 , decorrelation was caused by shadowing and attenuation effects underneath the hot ablation zone, as seen in the Bmode image. Fortunately, decorrelation artifacts can be detected from the normalized correlation map associated with the displacement image. Figure 3 shows a typical color-coded correlation image, where dark-red indicates perfect correlation (unity) and dark-blue stands for perfect decorrelation (zero). In the same figure, there are four different binary

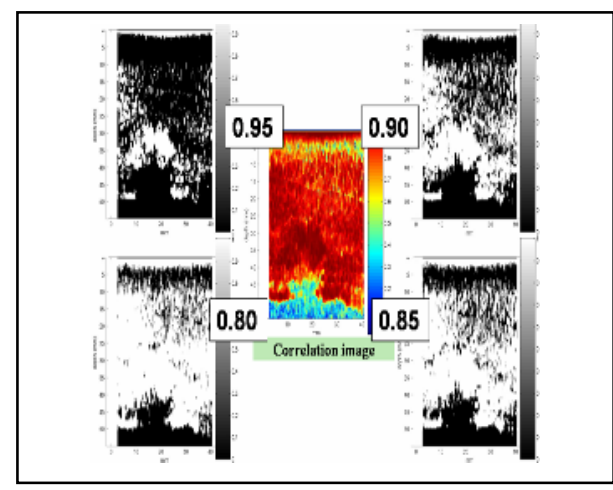

Fig. 3. Correlation in the displacement map, thresholded at different correlation levels

maps based on thresholds $0.95,0.90,0.85$, and 0.80 , all showing the spatial coverage of these correlation regions. The white region associated with correlations greater or equal to 0.95 is small compared to the region associated with correlations 0.80 . knowing the locations of better correlated regions helps us formulate a better conditioned shape optimization framework as shown in section 3.3.

\subsection{Theoretical Displacement Estimation}

To calculate theoretical displacements, a tissue elasticity model needs to be considered. We assume linear elastic, homogeneous and isotropic material. Moreover, liver tissues as mainly filled with water are incompressible, and hence Poisson's ratio is nearly 0.5 . We also apply uniform pressure by the transducer face in a quasi-static form, in which the deformation is considered plane strain. It is a type of deformation where there are no displacements in the z-direction, and the displacements in the $\mathrm{x}$ and $y$-directions are functions of $\mathrm{x}$ and $\mathrm{y}$ only. For plain strain problem, the assumption is $\varepsilon_{\mathrm{z}}=\varepsilon_{\mathrm{zx}}=\varepsilon_{\mathrm{zy}}=0$. Given a boundary conditions and a finite element mesh, the theoretical displacements $\mathbf{u}=(u, v)$ can be calculated by solving the following Navier's equations:

$$
\rho \frac{\partial^{2} u}{\partial t^{2}}-\nabla \bullet c \nabla u=K
$$

where $\rho$ is the material density, $\mathrm{K}$ are the body forces, and $\mathrm{c}$ is a tensor where each entry is a function of $G$ (shear modulus), $E$ (Young's modulus), and $v$ (Poisson's ratio).

\subsection{Shape Optimization}

Here we compare observed tissue displacements with simulated displacements using correlation map as weighting criterion. This weighting method puts high premium on the "most trusted" displacement areas and drives the evaluation of the domain points in the mesh at the corresponding locations. This approach reduces the resources needed for updating the mesh and effectively utilizes the available displacement information 
with minimal redundancy. Next, in an iterative optimization cycle where lesion location and shape parameters $S$, we adjust this hypothetical displacement field until it fits the actual displacement field. When the two fields are sufficiently similar, then the deformed model will yield the contours of the lesion. Thus, this inverse framework is trying to solve a non-linear optimization problem with the objective function:

$$
\widehat{S}=\arg \min \left\{\mathfrak{I}(S)=\sum_{i=1}^{N} \sum_{j=1}^{M} W(i, j)\|\widehat{u}(i, j)-u(i, j ; S)\|^{L 1}\right\}
$$

where $\widehat{S}$ is the estimated shape parameters, $\widehat{u}$ is estimated tissue motion, $\mathfrak{I}(S)$ is the objective function, $N, M$ are the number of rows and columns respectively in the displacement maps, and $W$ is the correlation map that acts as a weighing function to minimize the effects of distrusted tissue displacements. The experimental implementation is currently utilizing a modified version of the simplex search algorithm and LM method.

\section{Experiments and Results}

A thorough simulation study was conducted independently of any US imaging. The study involves running the finite element model to generate a displacement map where we know the ground truth shape parameters coupled to this map. Then, we use this displacement as the simulated input from tissue deformation. We have 5 parameters to optimize, ellipse location $(x, y)$, size $(a, b)$, and orientation. We tested the robustness of the method under different conditions and using different optimization schemes. The tests include partitioned optimization by solving $x, y$ together or separately, then solving for size parameters and orientation. This is performed while applying different objective functions and different search constraints.

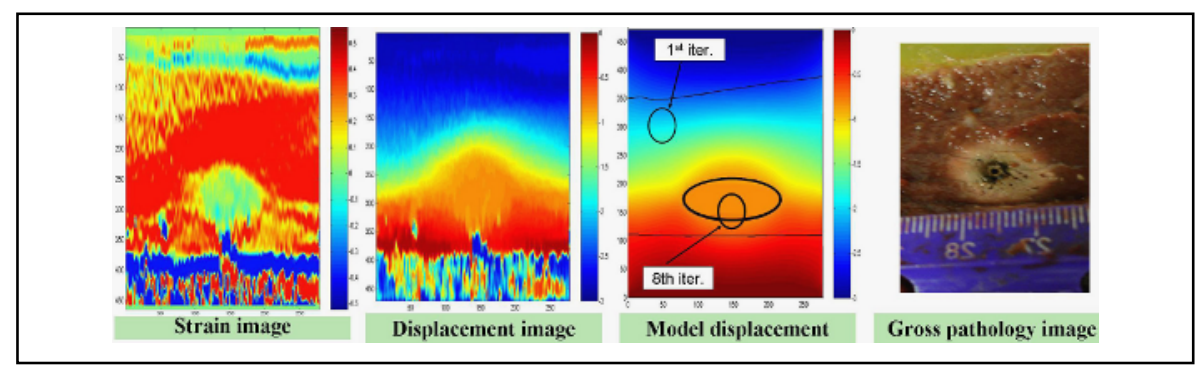

Fig. 4. Shows both strain and displacement images that reflects tissue deformations. Model displacement image represents FEM theoretical deformation. Also it illustrates a small circle to the top, left corner as an initial guess and another small circle after 8 iterations approaching to the final ellipse. Gross pathology picture is included to the right

The second study was conducted using a robotic ultrasound acquisition system. We used a Siemens Antares US scanner (Siemens Medical Solutions USA, Inc. Ultrasound Division, Issaquah, WA) with an ultrasound research interface (URI) to access raw RF data. A Siemens VF 10-5 linear array was used to acquire data. The tracking 
beams were standard B-mode pulses (6.67 MHz center frequency, F/1.5 focal configuration, apodized, pulse repetition frequency (PRF) of $10.6 \mathrm{KHz}$, with a pulse length of $0.3 \mu \mathrm{s})$. We collected data immediately after ablation of 18 ex-vivo fresh bovine liver samples; they are divided into three groups, 4min, 6min, and $8 \mathrm{~min}$ ablations using a Radionics device. All samples were soaked in degassed water to remove air pockets, and then embedded into gel material to support the liver during ablation and also maintain the assumed incompressibility of living tissues.

Figure 4 reflects the application of our approach on one ex-vivo liver sample ablated for $8 \mathrm{~min}$. Both strain and axial displacement from tissue deformation are included in the figure. The strain image shows good indication for the ablation zone. The FEM theoretical axial displacement at the last iteration has a good agreement with the corresponding tissue displacement. Also, we included the initial ellipse at the top-left corner, and in 8 iterations it becomes closer to the final shape, which was estimated in 15 more iterations. To evaluate the resulting segmentation, we use two independent ground truths. The first one deals with ellipse location, by relying on the ablator's tip location in the B-mode image before ablation. We have excellent agreement within $1 \mathrm{~mm}$ laterally. It is hard, however, to get a corresponding location axially, due to the unmeasured axial motion of the US probe before collecting strain data. The second ground truth deals with ellipse size, by using gross pathology picture to get an accurate measure for the ellipse size. In axial direction, our approach suggests 76.1 pixels $(10.49 \mathrm{~mm})$ where pathology picture gives about $11 \mathrm{~mm}$. In lateral direction, however, our approach numbers is 140 pixels $(18 \mathrm{~mm})$ where pathology is 15 $\mathrm{mm}$. This suggests that the axial displacement is very accurate in locating axial features but we probably need to regularize the objective function with lateral displacement estimation. Figures 5 and 6 present a convergence study for the scale parameters and center location, respectively. In both figures and for all four parameters, 10 iterations were enough to lock on a value with $10^{-4}$ difference variations.

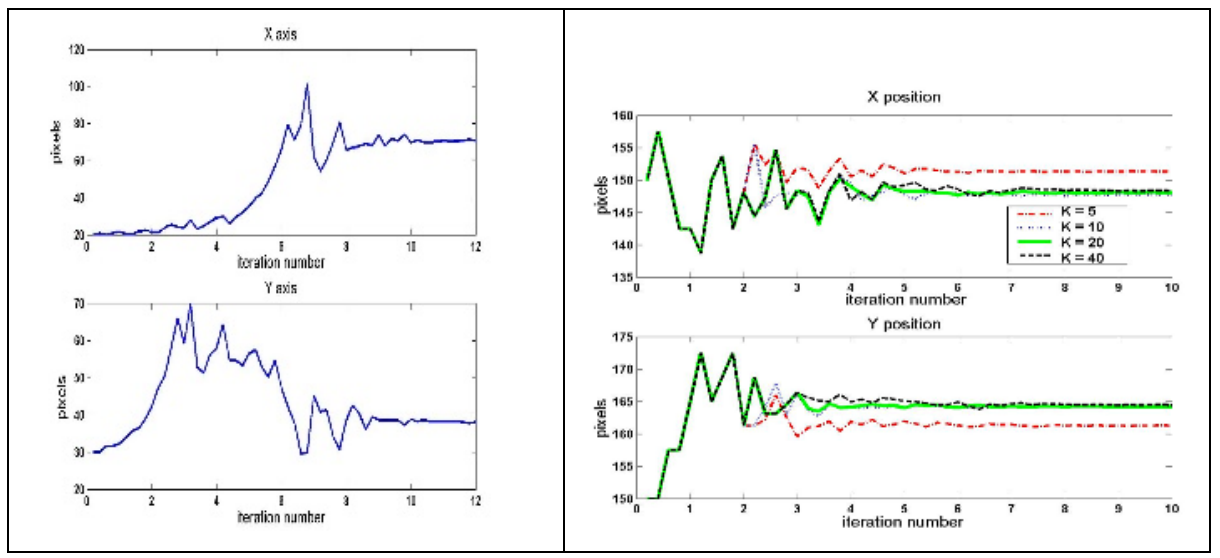

Fig. 5. Scale convergence of the ellipse

Fig. 6. Location convergence of the ellipse. It also shows the effects of $\mathrm{K}$ on optimizing shape parameters. $\mathrm{K}$ is the ratio of Young's modulus of ablated lesion to normal liver. 
We chose a suggested literature value of $1.5 \mathrm{KPa}$ for the Young's modulus of bovine liver, and tested the convergence of the FEM model to the center location using values of the Young's modulus for the ablated tissue, at 7.5, 15, 30, and $60 \mathrm{KPa}$. The results are shown in Figure 6, where the $\mathrm{K}$ parameter refers to the ratio of the ablated tissue, at 7.5, 15, 30, and $60 \mathrm{KPa}$. The results are shown in Figure 6, where the $\mathrm{K}$ parameter refers to the ratio of insensitive to variations for $\mathrm{K}$ values in the 10-40 range, in other words, regardless how hard we have cooked the tissue or how poorly estimate its Young's modulus, the model robustly converges. Also when we picked an unreasonable value, such as $\mathrm{K}=5$, the shape optimization algorithm was biased with only 35 pixels $(0.7 \mathrm{~mm})$.

\section{Conclusions}

We have developed and tested a novel shape optimization approach based on tissue deformation and shape priors. The results for the 18 samples will be reported in a detailed journal publication. Extending this framework to $3 \mathrm{D}$ is the next step for this project, which promises to increase the amount of measurements, while reducing the number of unknowns to just 9 parameters for an ellipsoid. Finally, the authors acknowledge the financial support from the NSF \#EEC 9731478 and Siemens Corporate Research.

\section{References}

1. Nakakura EK, Choti MA: Oncology (Huntingt). 2000 Jul;14(7):1085-98;

2. Buscarini L, Rossi S: Semin Laparosc Surg 1997;4:96-101.

3. Graham SJ, Stanisz GJ, Kecojevic A et al.: Magn Reson Med 1999;42(6):1061-71.

4. Kallel F, Stafford RJ, Price RE et al.: US. Med \& Biol., Vol. 25 (7), pp. 1099-1113, 1999

5. Bilgen M, Insana MF: J. Acoust. Soc. of Am., 101:1147-1154, 1997.

6. Ramamurthy BS, Trahey GE: Ultrasonic Imaging, 13:252-268 1991.

7. Chaturvedi P, Insana MF, Hall TJ: IEEE Trans. Ultrason. Ferroelec. Freq. Control, 45:179-191, 1998.

8. Kallel F, Ophir J: Ultrasonic Imag., 19:195-208, 1997.

9. O'Donnell M, Skovoroda AR, Shapo BM, Emelianov SY: IEEE Transactions on Ultrasonics, Ferroelectrics, and Frequency Control, 41:314-325, 1994.

10. Doyley MM, “An investigation into methods for improving the clinical usefulness of elasticity imaging." 1999. Institute of Cancer research, University of London. Thesis.

11. Ophir J, Cespedes I, Ponnekanti H et al.: Ultras. Imaging. 1991 Apr;13(2):111-34.

12. Varghese T, Ophir J: IEEE Trans. Ultrason. Ferroel. Freq. Cont., pp.164-172, 1997.

13. Varghese T, Techavipoo U, Liu W et al.: AJR Am J Roentgenol. 2003 Sep;181(3):701-7.

14. Curiel L, Souchon R, Rouvière O et al., "Elastography for the follow-up high-intensity focused ultrasound prostate cancer treatment" Ultrasound Med Biol. 2005 Nov ;31:1461-8

15. Kolen A, "Elasticity imaging for monitoring thermal ablation in liver" 2004, Institute of Cancer research, University of London. Thesis.

16. Varghese T, Techavipoo U, Zagzebski JA et al.: J Ultrasound Med. 2004 Apr; 23(4):53544; quiz 545-6. 